\title{
Beyond the giga-seal
}

\section{from Robert McBurney}

Two recent technical developments promise a new era in the examination of the movements of ions across the surface membranes of excitable cells. As well as the achievement of much higher current resolutions it is expected that one technique, using an 'excised patch', will make it possible to monitor changes in membrane properties while being able to directly influence the environment on the inside surface of the cell membrane.

The new techniques stem from the development by Neher and Sakmann (Nature 286, 71; 1980) of a method for the direct measurement of the ionic current flowing through single ion channels in biological membranes. These recordings of picoampere $\left(10^{-12} \mathrm{~A}\right)$ currents are made with a specially constructed glass pipette which seals off a small patch of membrane (about $\mathbf{1} \mu \mathrm{m}$ in diameter) on the surface of a cell from the rest of the surface membrane. The successful interaction of agonist molecules in the solution inside the pipette with receptor-ion channel complexes on the patch of membrane generates discrete pulses of ionic current which are recorded by a low-noise current-to-voltage converter. The current loop for measurements is from the pipette internal solution through open ion channels in the patch to the cytoplasm, then through 'leakage' channels in the membrane outside the patch to the bulk bathing solution and through the recording device to the pipette solution.

If some of the current flowing through the ion channels fails to complete this recording circuit, and a proportion returns via the seal between the pipette wall and membrane or through leakage channels in the patch itself, the rrue single-channel current amplitude is not measured. While the latter return pathway is not significant, except for larger patches on very small cells, the achievement of adequate seals has been a key factor in the application of this technique to different preparations. Not only does the relationship between seal resistance $\left(R_{\text {seal }}\right)$ and recording system resistance determine the proportion of the actual current recorded but the thermal current noise in the seal resistance (inversely proportional to $\sqrt{ } R_{\text {seal }}$ ) determines the lower limit of current resolution of the recording system.

Despite problems in creating adequate seals, the patch recording technique has been successfully applied over the past five years to a variety of preparations, including denervated frog muscle, cultured rat muscle (myoballs), locust muscle and

Robert McBurney is in the Department of Physiology, The Medical School, Newcastle upon Tyne. squid giant axons. The channels investigated have mostly been those activated by receptor agonists (cholinergic in frog and rat muscle; glutaminergic in locust muscle), although in one case (squid giant axon) the properties of voltagedependent $\mathrm{K}^{+}$channels have been studied. The technique has been used to confirm and extend data obtained by the analysis of membrane current fluctuations (see Stevens Nature 270, 391; 1977). In addition, it has yielded new information concerning the detailed kinetics of action of some drugs, for example local anaesthetics on acetylcholine-activated ion channels (Neher and Steinbach J. Physiol, Lond. 277, 153; 1978), and has revealed the behaviour of ion channels during desensitization (Sakmann et al. Nature 286, 71; 1980).

The first exciting new development to come from Neher and Sakmann's technique arose from efforts to increase the sealing resistance. By applying suction to the recording pipette as it was placed on the surface membrane of a cultured muscle cell, F. Sigworth and E. Neher (Göttingen) found that a very high-resistance seal $\left(>10^{9} \Omega\right)$ was, in some cases, suddenly created. This seal and its associated low current-noise even at a bandwidth from DC-7 $\mathrm{kHz}$ allowed Sigworth and Neher to resolve single voltage-dependent $\mathrm{Na}^{+}$ channels: those normally associated with the generation of muscle action potentials. In their preliminary report (Nature 187, $447 ; 1980$ ), as well as describing the 'gigaseal' and presenting clear records of single $\mathrm{Na}^{+}$channels, they have shown that these channels have conductance and average lifetime values which agree with those obtained by more indirect means. They also demonstrate the independence of channel behaviour by showing that the sum of many records, usually containing just one channel opening in response to a fixed membrane depolarization, mimics the behaviour of many channels operating simultaneously.

The future application of the 'giga-seal' technique to excitable tissues will permit the investigation of 'gated' ion channels in cells or regions of cells not amenable to voltage-clamp analysis with intracellular microelectrodes, such as smooth cells, gland cells, unmyelinated axons and possibly even nerve terminals. In addition, the technique provides improved current resolution and, for single-channel currents already observed by the original method, a much wider bandwidth for recording which will undoubtedly reveal finer details in the kinetics of channel activation and inactivation.

Perhaps more exciting than the achievement of the 'giga-seal' itself has been the recent observation made by two groups of workers, R.Horn and J. Patlak (Yale) and O. Hammill and B. Sakmann (Göttingen). They found that, once established, this seal was almost impossible to shake off. When the electrode was pulled away from the cell it seemed that the patch of membrane was excised on, or within, the tip of the electrode and singlechannel currents could still be observed if the transmembrane voltage was appropriately adjusted. After a short time the recording ceased, possibly due to a membrane-bound vesicle being formed within the electrode tip (because the highresistance seal remained). The two groups solved the problem of vesicle formation in different ways: Horn and Patlak (Proc. natn. Acad. Sci. U.S.A. 77, 6930; 1980) found that fluoride ions delayed or prevented the process; while Hamill and Sakmann (J. Physiol., Lond. in the press) found that by lifting the electrode through an air-water interface they were able to reexpose the patch.

In their preliminary report Horn and Patlak illustrate one of the special features of this preparation: they examine the dependence of the conductance of acetylcholine-activated channels on sodium ion concentration in a situation where the latter could be defined on both sides of the membrane. Such access permits a vast array of experiments to be undertaken with controlled environments on either side of the membrane, similar in principle to the study of ion transport in 'doped' artificial lipid membranes, and these will certainly result in a clearer idea of the mechanism of ion transport and selectivity of natural ionophores.

However, the most important aspect of the preparation mav simply prove to be access to the inside surface of cell membranes. It will now be possible to investigate directly the action of intracellular messengers (for example, cyclic nucleotides, $\mathrm{Ca}^{2+}$ ) and cellular metabolic products on membrane excitability and transport processes. It is hoped that this 'excised patch' technique will be generally applicable to cells derived from a wide range of tissues. For example, its application to heart cells will certainly provide an excellent preparation with which to study the relationships between 'second messengers', and protein phosphorylation and ion channels in the hormonal modulation of membrane conductance changes underlying cardiac pacemaker activity.

Erratum: In Antimatter back to front by C. $H$. Llewellyn Smith (Nature 289, 534 February 12, 1981) the last sentence in the first paragraph should have read "The discovery of CP violation by Christenson, Cronin, Fitch and Turlay in 1964, for which Cronin and Fitch were awarded the 1980 Nobel prize, was therefore almost as great a shock as the discovery of parity violation".

Corrigendum: In Cement - a respectable material? by D. D. Double $(289,348$, January 29,1891$)$ the figure of " $100 \mathrm{MN} \mathrm{m}^{-2}$ " quoted as the modulus of mother-of-pearl shell should have been "about 60 $\mathrm{GN} \mathrm{m}{ }^{-2} "$. 